\title{
REVIEWS
}

\section{TWIN STUDIES OF SCHIZOPHRENIA}

HEREDITY AND ENVIRONMENT IN THE FUNCTIONAL PSYCHOSES. E. Kringlen, M.D. 200 pp. Published by Universitetforlaget, Oslo. 1967.

Schizophrenia is one of the major curses of man. It has been shown in a number of European countries that nearly 1 in 100 individuals develop the condition at some stage in their lives and a similar incidence has been reported in Japan. Variation occurs in incidence by social class, but this probably represents only a tendency for those affected to move down the social scale. Modern treatment has greatly reduced the time spent in hospital with schizophrenia, but an attack is still usually followed by permanent changes in personality.

Views on the aetiology of the condition range from the purely environmental hypotheses of many American psychoanalysts to the purely genetic hypotheses of certain continental psychiatrists. The evidence for a genetic element in causation comes from family studies: the similar (approximately 10 per cent.) risk to sibs and children; the similar risk to children whether it is the mother or the father who is schizophrenic; the raised risk to children (to about 40 per cent.) where both parents are schizophrenic, but not where one is schizophrenic and the other psychopathic; the preliminary indications that the risk to children remains about 1 in 10 even when the child is separated from the mother at birth; the three or four times greater risk to monozygotic than to dizygotic co-twins of patients with schizophrenia; the preliminary indications that the risk to monozygotic co-twins remains high when the twins were separated at birth.

Dr Kringlen's monograph describes a good example of a twin study, combined with a family study. He started with a twin register for Norway from 1901 to 1930 and checked this against the register for psychotic patients. The records of men and women who were on both registers were examined, the diagnoses checked, the patients traced and examined, and the type of twinning established. The largest group were the schizophrenics; the manic-depressive and reactive psychotic patients were too few to provide much information.

For schizophrenia the straight twin concordance rates were (without a double count where both members of the pair were index patients and without age correction) for monozygotic pairs 14 in 55 (25 per cent.) and for like sex dizygotic pairs 6 in 90 (7 per cent.). Some of the monozygotic co-twins were schizophrenic, although not on the register, and this raised the concordance figures to 38 per cent. for monozygotic and 7 per cent. for dizygotic pairs. There was a trend to greater concordance for monozygotic pairs when the illness was more severe. Most of the earlier studies, based on hospital series, have given a higher concordance rate (about 60 per cent.) for monozygotic twins. However, Dr Kringlen's method of ascertainment was such that members of concordant pairs would usually have been independently ascertained. In terms of the most important statistic, the proportion of co-twins of index patients also affected. this series from Norway is compatible with other studies. 
Dr Kringlen notes that his findings support the view that there is a considerable genetic component in the causation of schizophrenia. He considers it most unlikely that the greater twin concordance can be entirely explained by a more similar environment of the monozygotic pairs, and searched, without much success, for environmental factors affecting concordance. The series included three of the especially valuable monozygotic pairs separated in infancy; of these one were concordant and two discordant. The concordant pair were separated at three months of age, but were brought up in rather similar homes.

As to the nature of the predisposing genetic factors, Dr Kringlen favours a polygenic hypothesis. With a common condition, the distinction between this hypothesis and that of a single gene, with incomplete manifestation in the heterozygote, is not easily made. The distinction is of practical importance since, if a single gene is of major importance, research should be directed at finding a single specific biochemical error.

Twins and family studies of this kind have perhaps given almost all the information they can. Efforts should now be concentrated on studies of genetic-environmental interaction in separated twins, adopted children and especially perhaps on detailed prospective studies of the offspring of two schizophrenic parents; as well as on biochemical studies.

\section{O. Carter \\ Medical Research Council Institute of Child Health} PROCEEDINGS OF THE THIRD INTERNATIONAL CONGRESS OF HUMAN GENETICS.
Ed. J. F. Crow, and J. V. Neel. The Johns Hopkins Press, Baltimore. pp. $v+578$. E6. $18 \mathrm{~s}$.

This volume is the published account of the Plenary Sessions and Symposia of the Third International Congress of Human Genetics held at Chicago from 5 th to 10th September 1966. Like all such published accounts it contains a huge amount of information of extremely problematical value to the geneticist. For example, the Minutes of the Business Meeting and the Statutes of the Permanent Committee are only likely to appeal to a minority. Similarly, the address given to the Plenary Session by H. J. Muller, although an interesting account of his views, is scientifically almost valueless since he has seen fit not to back up his views with adequate references. He specifically leaves out all references since he claims that to include them would require " drawing invidious lines between contributors to the background material". It is a general weakness of Congresses that distinguished men are often prevailed upon to speak at a time when they have very little of value to contribute. This in itself would not matter except that their contributions are subsequently published. Leaving aside the Plenary Sessions and the public lectures, in which only Penrose gave an adequate reference list, the organised Symposia show a great unevenness in quality. Some are exceptionally good and informative, others contain papers which would have hardly gained space in any journal which employs referees.

The production of this book is of a very high quality and is well edited, although perhaps some of the figures such as part of a Rhesus code in situ on the Rolodex file could have been omitted without serious loss. There are also some apparent misprints. For example, the statement on page 392 